Das opus magnum des mittlerweile im schottischen Aberdeen lehrenden Verfassungsjuristen beleuchtet mit einem methodisch breit ausgebildeten Instrumentarium letztlich die einschlägigen Steuerungsdilemmata sich immer weiter funktional differenzierender Gesellschaften, aber eine wirkliche Lösung chronischer Staatsüberforderung kann auch er nicht bieten. Am ehesten sei noch das deutsche Staatskirchenrecht als ein archetypisches Referenzgebiet ausgewiesen. Aber gerade der Charakter von Konkordaten als völkerrechtlichen Verträgen, die mit dem Vatikan geschlossen werden, unterscheidet sich kategorial von Abreden, die mit Privaten oder auch mit Selbstverwaltungsorganen betroffener Partikularinteressen hinsichtlich künftiger Gesetzgebung getroffen werden.

Politisches Recht beschreibt ein äußerst fluides Medium: Nur wenige Monate, nachdem das Buch von Becker erschienen war, sollte sich bekanntlich die Frage stellen, was aus dieser im Einklang mit Betroffenen vertraglich vereinbarten Politik unter der neuen Regierung werden sollte. Nicht nur die Große Koalition, sondern auch die Vertreter der Partikularinteressen müssen sich angesichts der Langfristigkeit der getroffenen Regelungen entscheiden, welches weitere Vorgehen ihnen mehr nützt. Somit wird vor allem das Problem der Kernenergie bislang weitgehend dilatorisch behandelt. Es könnte sich als weitsichtiger erweisen, die Vereinbarungen der Vorgängerregierung unangetastet zu lassen, als auf eine Revision des Konsenses zu pochen. Was das Rechtliche anbetrifft, so votiert Becker ohnehin für eine Selbstbindung der Politik, die sich im Zweifel selbst auf das Parlament erstrecken soll. Die Kernenergie ist hierbei nur Menetekel zukünftiger Probleme von Technik und Forschung, die immer stärker Eigendynamik und Eigengesetzlichkeit in ihrer zeitlichen Planung folgen und sich nicht in das Prokrustesbett von Legislaturperioden pressen lassen. Droht technisch-wissenschaftlicher Fortschritt, mit demokratischer Staatsverfassung gar unverträglich zu werden?

Ob der kooperative Staat als verheißungsvolle via regia zu einer Renaissance staatlicher Steuerung führen oder als neuer Nachtwächterstaat einen trüben Holzweg in eine entstaatlichte Postmoderne darstellen wird, bleibt indes das Geheimnis der Zukunft.

Daniel Hildebrand

\title{
Das neue deutsche Regierungssystem
}

Sturm, Roland und Heinrich Pehle: Das neue deutsche Regierungssystem. Die Europäisierung von Institutionen, Entscheidungsprozessen und Politikfeldern in der Bundesrepublik Deutschland, 2., aktualisierte und erweiterte Auflage, VS Verlag für Sozialwissenschaften, Wiesbaden 2005, 373 Seiten, $€ 19,90$.

Es gehört heute zum Bestand gesicherter politikwissenschaftlicher Erkenntnis, dass die Europäische Union einen immer stärkeren Einfluss auf nationalstaatliche Politikprozesse ausübt. Durch eine stetig wachsende Zahl europäischer Richtlinien und Verordnungen, die in nationales Recht überführt werden müssen, hat die EU eine erhebliche agenda settingMacht bekommen. Daher ist der Begriff der Europäisierung zu einem in der Politikwissenschaft gängigen, allerdings auch immer unbestimmteren Konzept geworden, das die fort- 
schreitende Wechselwirkung zwischen supranational-europäischen und nationalstaatlichen institutionellen Arrangements beschreibt. Dazu haben Roland Sturm und Heinrich Pehle ein Buch - mittlerweile in zweiter und erweiterter Auflage - vorgelegt, das sich an der Schnittstelle zwischen einer Einführung in das politische System der Bundesrepublik und einer Institutionenkunde der EU verorten lässt. Während die überwiegende Zahl der Darstellungen zum politischen System der Bundesrepublik auf die Innenwelt konzentriert bleibt, bietet der vorliegende Band, wie die Autoren im Vorwort zu Recht betonen, eine „ergänzende Sichtweise im Hinblick auf die Einführungsliteratur zum deutschen Regierungssystem“ (S. 6). Der Untertitel macht deutlich, dass es sich um eine klassische aus der Politikfeldforschung stammende Herangehensweise handelt. So legen die Autoren gleichsam über alle wesentlichen Aspekte des policy making die Folie der Europäisierung und beleuchten so den Einfluss europäischer Politik auf Institutionen (polity), Entscheidungsprozesse (politics) und Politikfelder (policy) in Deutschland.

Aufgeteilt in sieben Kapitel gliedert sich das Buch zunächst in zwei große Abschnitte, von denen der erste die Wirkungen europäischer Politik auf die wesentlichen Verfassungsorgane (Bundesrat, Bundestag, Bundesregierung u.a.) sowie auf politische Prozesse thematisiert. Der zweite große Teil folgt spezifischen Politikfeldern, zum Beispiel Agrar-, Umweltoder Währungspolitik.

Trotz der häufigen Verwendung des Konzeptes der Europäisierung bleibt dieser ubiquitär verwendete Begriff allzu oft unscharf. Sturm und Pehle stellen ihren Ausführungen jedoch eine umfassende und präzise Definition voran. Demnach ist Europäisierung „ein politisch-gesellschaftlicher Prozess, der angetrieben von der Geschwindigkeit und Reichweite der europäischen Integration einen Veränderungsdruck auf Nationalstaaten und europäische Gesellschaften ausübt, aber auch europäische Institutionen zur Responsivität gegenüber nationalen Interessen zwingt und damit diese zu fortwährendem politischen Wandel und zum Teil auch zu institutioneller Anpassung bewegt" (S. 12). Bedauerlicherweise werden dann jedoch weitergehende definitorische Feinheiten in eine Fußnote verlagert. Gerade hier wäre es wünschenswert gewesen, diese Diskussion im Text des Einleitungskapitels zu lesen. Unabhängig jedoch von dem Versuch, Europäisierung als Konzept möglichst genau zu erfassen, bildet der Teil über die Auswirkungen auf Institutionen und Gesellschaft des deutschen Nationalstaates eine fruchtbare Basis für das gesamte Buch. Hier werden unterschiedliche theoretische Zugänge vorgestellt, auf die sich die später folgende Diskussion empirischer Ergebnisse bezieht. Die Wechselbeziehung und die Integration zwischen europäischer und nationalstaatlicher Ebene erhalten durch Konzepte wie Politikverflechtung, Netzwerke, Rücknahme von Staatlichkeit (als so genannte negative Integration) ihren theoretischen Rahmen.

Während der Titel des Buches ankündigungsstark vom „neuen“ Regierungssystem spricht und Sturm und Pehle eine „fundamentale Neugestaltung“ (S. 12) der politischen Institutionen diagnostizieren, bleibt nach dem innovatorischen Gehalt zu fragen. Im Mittelpunkt steht nicht die Entstehung eines neuen europäischen Staatengebildes, sondern nach Aussage der Autoren - die Veränderung des politischen Systems der Bundesrepublik im Lichte fortschreitender Europäisierung. Damit wird allerdings eine Verengung auf den erst genannten Aspekt (wachsender Druck auf Nationalstaaten) der zuvor angebotenen Europäisierungsdefinition vorgenommen.

Das Hervorheben von Europäisierungstendenzen in Policy-Bereichen wie Umwelt-, Wettbewerbs- oder Verkehrspolitik im zweiten großen Abschnitt ist zunächst einleuchtend, 
sind doch besonders auf diesen Gebieten europaweite Regelungen zu beobachten. Im verschachtelten Mehrebenensystem der EU hat der sich laufend vollziehende Souveränitätsund Kompetenztransfer „nach Europa“ unter anderem dazu geführt, dass nationale Regierungen mit Hilfe der europäischen Ebene „über Bande“ spielen. Unter Verweis auf europäische Harmonisierungserfordernisse können unpopuläre Politiken auf der nationalen Ebene zum Teil leichter durchgesetzt und so eine „Reduktion von Legitimationskosten im nationalen Rahmen“ (S. 209) erreicht werden. Das Kapitel über die Europäisierung einzelner Politiksektoren wäre insgesamt vollständiger, wenn zudem die Herausbildung der Gemeinsamen Außen- und Sicherheitspolitik der EU thematisiert worden wäre.

Während der Titel programmatisch ein „neues“ Regierungssystem annonciert, „bedeutet dies nicht“, so Sturm und Pehle im konzisen Schlusskapitel einschränkend, „dass schon heute eindeutig zu bestimmen ist, wie das ,neue' deutsche Regierungssystem an einem fiktiven Ende des Europäisierungsprozesses aussehen wird“ (S. 359). Auf diese Weise rücken die Autoren schließlich ein Stück von ihrer selbst gegebenen Einschätzung einer Neugestaltung des Regierungssystems ab.

Insgesamt haben Sturm und Pehle ein sehr informatives, klar strukturiertes und gut lesbares Buch vorgelegt. Geschrieben aus einer doppelten Perspektive ist es ohne Zweifel für einen breiten Leserkreis geeignet. Zum einem bietet es umfassende Einsichten in die vom deutschen Regierungssystem zu erbringenden Anpassungsleistungen als Folge sich beschleunigender Integrationsfortschritte in Europa. Zum anderen beleuchtet der Band viele Aspekte der europäischen Integration und wirft damit ein sehr helles Licht auf das, was oft nur sehr halbherzig und ebenso unzutreffend als „Brüsseler Politik“ apostrophiert wird.

Helge F. Jani

\section{Föderalismus in kleiner Münze}

Bretz, Kay G.: Föderalismus und Regionalismus in Deutschland, Spanien und der Europäischen Union, Verlag Duncker \& Humblot, Berlin 2005, 450 Seiten, € 86,80.

Mit Charakteristika und Zusammenhängen von Föderalismus und Regionalismus im deutschen Bundesstaat und Spaniens „Staat der Autonomien“ beschreibt Kay G. Bretz mit sehr eigener Terminologie Europas Staatsstrukturprinzipien. Er prüft, ob ein vom Unionsvolk begründeter Unionsstaat vereinbar ist mit den Verfassungsprinzipien. Unabdingbar seien der Staat als „Vereinigung einer Menge Menschen unter Rechtsgesetzen“ (Kant), die Republik und eine kleine Einheit „als menschenrechtliches Prinzip zur Persönlichkeits- und Freiheitsentfaltung der Menschen" (S. 49). Es fragt sich sofort: Was ist mit den Monarchien in der EU? Und: Die kleine Einheit als sine qua non der Freiheit - die England oder Frankreich mangels verfassungsrechtlicher Subsidiarität nicht kennen? Da die Erosion von Legislativfunktionen der Länder an die Grenze von Art. 79 Abs. 3 GG stoße, gefährdet Europas Integration für Bretz den Föderalismus - wie schon für seinen Doktorvater Schachtschneider, der gegen den Maastricht-Vertrag und den Euro klagte. Ungeprüft setzt er den deutschen Föderalismus gleich mit Österreichs Konglomerat früherer Kronländer und Belgiens sich überlappenden sprachlichen und territorialen Regionen. Die Gliedstaaten der EU seien 